\title{
La Terapia de Aceptación y Compromiso como herramienta para optimizar las intervenciones potenciadoras del envejecimiento activo
}

\author{
María Márquez-González, Virginia Fernández-Fernández ${ }^{2}$, Rosa Romero-Moreno ${ }^{2}$ y \\ Andrés Losada ${ }^{2}$ \\ 'Departamento de Psicología Biológica y de la Salud. Facultad de Psicología. Universidad Autónoma de Madrid. \\ ${ }^{2}$ Departamento de Psicología. Facultad de Ciencias de la Salud. Universidad Rey Juan Carlos.
}

En el presente trabajo se plantea una reflexión en torno a la gran consistencia y potencial complementariedad existente entre la perspectiva de la intervención clínica, especialmente la cada vez más popular terapia de aceptación y compromiso (ACT), y los modelos teóricos en los que se ha sustentado la perspectiva del envejecimiento activo (perspectiva del ciclo vital). Se propone que la terapia ACT puede contribuir a la optimización del repertorio de herramientas y estrategias para potenciar el envejecimiento con éxito. Se revisan brevemente los modelos de desarrollo a lo largo del ciclo vital, haciendo énfasis en cómo éstos se articulan en torno a los constructos "meta" y "acción" y subrayan el papel de los procesos de autorregulación y ajuste flexible de metas en la adaptación a lo largo del ciclo vital. Finalmente, se describe brevemente una propuesta concreta de intervención basada en la terapia ACT actualmente en desarrollo, dirigida a personas que cuidan de familiares mayores con demencia.

In this paper, it is proposed a reflection on the great consistency and potential complementarity between the perspective of clinical intervention, especially the progressively more popular Acceptance and Commitment Therapy (ACT), and the theoretical models in which the perspective of active/successful aging is grounded (life span theories). It is suggested that ACT can help optimize the repertoire of tools and strategies available to potentiate the active aging interventions. Theoretical models are briefly reviewed, with an emphasis on analyzing how these models builds upon the constructs of "goal" and "action", and highlight the main role of self-regulation and flexible goal adjustment for adaptation across the lifespan. Finally, a specific ACT-based intervention program currently in progress and directed to dementia family caregivers is outlined.

\section{nalabras clave/keywords:}

Terapia de Aceptación y Compromiso, envejecimiento activo o con éxito, perspectiva del ciclo vital, adaptación, cuidadores,

Acceptance and Commitment Therapy, active/successful aging, lifespan, adaptation, caregivers

\section{Agradecimientos}

La preparación de este artículo ha sido parcialmente financiada por el Ministerio de Ciencia e Innovación (PSI200908132) y por el Ministerio de Economía y Competitividad (PSI2012-31293). 


\section{Introducción}

Es un hecho constatado en nuestro país que el grupo de población formado por las personas mayores de 65 años continúa aumentando y que nuestra esperanza de vida (tanto al nacer como a los 65 años) es cada vez mayor (Abellán y Ayala, 2012). Además, el perfil biopsicosocial de la población mayor está cambiando, en lo que se refiere a las competencias, necesidades, actitudes e intereses que presentan estas personas. Por otro lado, cada vez es mayor la heterogeneidad entre las personas mayores de 65 años, dada la diversidad de las trayectorias de envejecimiento. El cambio en el perfil de la población de personas mayores impone importantes desafíos a la sociedad, en general, y a la intervención psicológica, en particular. Hasta hace relativamente poco tiempo y, en gran medida, todavía en la actualidad, la escasa "cultura psicológica" de las generaciones de personas mayores ha supuesto dificultades para la comprensión y asimilación de conceptos y procedimientos de la psicología como ciencia y profesión, así como para considerar "la cuestión psicológica" (emociones, pensamientos, comportamientos) como algo importante implicado en su bienestar y la calidad de vida. Debido a esta característica, asociada a factores de cohorte de naturaleza sociocultural e histórica, las personas mayores no han sido ni son grandes frecuentadores de los servicios de ayuda psicológica. Probablemente debido a esa distancia mantenida durante mucho tiempo por las personas mayores en relación a la ayuda psicológica, así como a la presencia de potentes estereotipos relacionados con la inevitabilidad del sufrimiento psicológico o con la desconfianza en la posibilidad de "cambio" en las personas mayores, el desarrollo de la disciplina de la intervención clínica con población mayor ha sido más lento y se encuentra, todavía en la actualidad, menos estructurado y formalizado que el tratamiento de problemas psicológicos en población adulta más joven. Sin embargo, es esperable que el cambio en el perfil de la población mayor conlleve importantes cambios en este sentido, siendo altamente probable que las nuevas generaciones de personas mayores acudan a la psicología en busca de ayuda para adaptarse a los problemas o circunstancias difíciles que les toque vivir, para mejorar su calidad de vida, desarrollar estilos de vida más saludables o envejecer de forma más activa o exitosa.

\subsection{Aportaciones de la intervención clínica psicológica para optimizar el envejecimiento activo}

Ante la situación del cambio de perfil de las personas mayores, cabe preguntarse si la ciencia psicológica tiene respuestas adecuadas para proporcionar ayuda psicológica eficaz a estas personas. En este sentido, existen intervenciones psicológicas empíricamente validadas para abordar problemas psicológicos concretos en las personas mayores. Concretamente, la terapia cognitivo-conductual (TCC), orientada al cambio de pensamientos y conductas para facilitar la adaptación de las personas a sus circunstancias, ha demostrado su eficacia para tratar fundamentalmente problemas de depresión, ansiedad, insomnio y dolor crónico en personas mayores (Gatz, 2007). Por otro lado, la mayoría de las personas mayores se puede beneficiar igualmente de intervenciones psicosociales centradas en la promoción de estilos de vida saludables y envejecimiento activo, así como en la prevención de la dependencia y otras situa- 
ciones indeseables (Fernández-Ballesteros, Caprara, Íñiguez y García, 2004).

Centrando la atención en la perspectiva del envejecimiento activo, puede constatarse que este paradigma de actuación, cuyas bases teóricas podrían encontrarse en la teoría de la actividad y la perspectiva del ciclo vital, se ha desarrollado de forma relativamente independiente a la disciplina de la intervención psicológica clínica en la vejez. Esto es perfectamente entendible, ya que el paradigma de envejecimiento activo o con éxito es más cercano conceptualmente a la perspectiva de bienestar eudaimónico y de la psicología positiva, centrada en la promoción del bienestar integral de la persona, que es distinto y va más allá de la disminución del malestar psicológico. El envejecimiento con éxito se ha definido como una "baja probabilidad de enfermedad y de la discapacidad asociada, alto funcionamiento cognitivo $\mathrm{y}$ capacidad física funcional y compromiso activo con la vida" (Rowe y Kahn, 1997; p.433). Asumiendo esta definición, en este trabajo planteamos que la perspectiva de la intervención psicológica clínica y su consideración y abordaje de factores psicológicos disfuncionales que explican el origen y mantenimiento de conductas desadaptativas (manifiestas y encubiertas), no sólo es compatible sino que puede enriquecer en gran medida la intervención dirigida a potenciar el envejecimiento con éxito. En este sentido, consideramos que la perspectiva de la intervención clínica ofrece herramientas que permiten abordar, en la evaluación e intervención psicológica, diversas dificultades, problemas o barreras de tipo psicológico o subjetivo que presentan algunas personas mayores y que limitan claramente o interfieren en su camino hacia un enveje- cimiento exitoso. Concretamente, patrones inflexibles de pensamiento como el pensamiento absolutista (p.ej. "cualquier tiempo pasado fue mejor...”), estereotipos sobre el envejecimiento (p.ej. "a esta edad ya no se puede esperar nada"), o rigidez en la selección e implicación en valores, metas y objetivos, suelen asociarse a dificultades para adaptarse a los cambios asociados al envejecimiento, que requieren de la persona que envejece un reajuste flexible de sus metas de cara a "seguir enganchadas en la vida" a través de acciones con significado. A menudo, esta inflexibilidad cognitiva y motivacional da lugar al desarrollo de patrones de comportamiento poco adaptativos (pasividad, evitación de situaciones o contextos, aislamiento) que interfieren y limitan la capacidad funcional de las personas y generan altos niveles de depresión, ansiedad o irritabilidad, disminuyendo la satisfacción con la vida y obstaculizando su envejecimiento activo o exitoso.

Como puede apreciarse, consideramos los constructos de "valor", "meta" y "acción con significado" y los procesos de autorregulación relacionados con éstos como aspectos implicados de forma fundamental en el bienestar y la satisfacción con la vida en la vejez. Algunos de estos aspectos (metas y acciones) son precisamente elementos centrales de los principales modelos teóricos que han servido de base a la perspectiva del envejecimiento con éxito. Sin embargo, puesto que tales modelos han sido propuestos desde un plano teórico más básico y no tanto desde la perspectiva clínica aplicada, no es sencillo deducir de ellos pautas de intervención concretas y operativizadas para manejar tales variables (metas y acciones) en el contexto de la intervención psicológica con las personas mayores. Es por ello 
que consideramos relevante reflexionar sobre cómo los modelos de intervención clínica pueden complementar a estos modelos teóricos, contribuyendo a clarificar pautas concretas de intervención terapéutica.

Específicamente, el enfoque terapéutico denominado Terapia de Aceptación y Compromiso (ACT, Hayes, Strosahl y Wilson, 1999; 2011), de gran popularidad en los últimos años, y que hace especial hincapié en la promoción de la activación conductal comprometida con los valores personales, así como en la aceptación de eventos externos e internos inmodificables, puede aportar elementos fundamentales que hasta ahora no se habían tenido en cuenta explícitamente en los modelos teóricos existentes en la literatura gerontológica. En este sentido, esta perspectiva teórica se está perfilando como una propuesta especialmente interesante para optimizar la intervención psicológica con esta población (MárquezGonzález, 2010; Petkus y Wetherell, 2013), ya que encaja plenamente con la perspectiva teórica del ciclo vital en cuanto a la centralidad conferida a la motivación y acción dirigida hacia metas relacionadas con los valores personales, así como al ajuste flexible de las mismas para conseguir la adaptación a las pérdidas y cambios asociados al envejecimiento (Alonso, López, Losada y González, 2013).

\section{Bases teóricas del envejecimiento activo: la autorregulación, el control y las metas como ejes de la adapta- ción a lo largo del ciclo vital}

Ya en los años 50, la Teoría de la Actividad de Havighurst y Allbrecht (1953) postuló que el mantenimiento de la actividad, la sustitución de roles perdidos por nuevos roles y el mantenimiento de la implicación psicológica en la sociedad, esto es, en la corriente de la vida, constituyen las claves fundamentales para un envejecimiento con éxito. Concretamente, esta perspectiva planteó que el éxito en la vejez depende del rendimiento de la persona en, al menos, cuatro dimensiones: 1) el nivel de actividad en el que se implica; 2) la capacidad para desengancharse o desconectarse de determinadas actividades o metas que ya no son alcanzables; 3) la satisfacción con la vida; y 4) la madurez o integración de la personalidad. Un modelo teórico que podría considerarse como una versión actualizada y enriquecida del modelo de la actividad es el paradigma teórico elaborado por Carol Ryff y colaboradores (p.ej., Ryff y Keyes, 1995), para quienes el bienestar en la vejez es entendido más como una integración generadora de sentido y significado de la propia vida y acciones de la persona (bienestar eudaimónico o psicológico), que como la mera ausencia de malestar y de emociones negativas o la promoción de emociones positivas y felicidad (perspectiva hedónicasubjetiva). Este modelo de bienestar está integrado por seis dimensiones distintas que presentan trayectorias evolutivas diferentes y que son: autoaceptación, dominio sobre el entorno, propósito y significado en la vida, crecimiento personal, relaciones positivas con otras personas y autonomía.

En los años 80 y principios de los 90 se consolida la perspectiva del ciclo vital, metamodelo teórico que cristalizó en el modelo de Optimización Selectiva con Compensación (SOC; Baltes y Baltes, 1990) y en el que se han fraguado formulaciones teóricas posteriores más concretas como la Teoría del Desarrollo Intencional (Brandtstädter y Greve, 1994) o la Teoría del Control a lo largo del Ciclo Vital (Heckhausen y Schulz, 1995). 


\subsection{El Modelo de Optimización Selecti- va con Compensación (SOC)}

Desde la perspectiva del ciclo vital y, más concretamente, desde el modelo SOC (Baltes y Baltes, 1990), se considera que los elementos integradores del proceso de desarrollo humano son la biología, la cultura y la propia persona en desarrollo, quien define sus cursos de acción y elecciones a través de sus objetivos y metas. Estos elementos, junto con otros factores asociados a la edad de la persona y su historia contextual y biográfica, interactúan entre sí para moldear la adaptación de la persona a los distintos momentos del ciclo vital.

Si bien se asume que, en cualquier momento del ciclo vital, el desarrollo implica tanto pérdidas como ganancias (relación dialéctica), también se considera que el envejecimiento trae asociadas importantes restricciones en los recursos tanto biológicos como sociales y personales, lo cual supone un incremento de las pérdidas respecto de las ganancias en esta fase del desarrollo. A pesar de las progresivas mermas y restricciones en sus recursos, es posible para la persona que envejece adaptarse a sus contextos vitales con éxito, lo cual implica necesariamente que continúe implicada en acciones que le permitan cumplir sus metas significativas, a pesar de los cambios. De acuerdo con el modelo SOC, mientras que en la juventud y vida adulta joven predominan las metas relacionadas con el crecimiento y expansión de los recursos (desarrollar nuevas capacidades, conocer personas nuevas, etc.), en la vejez las metas se centran en el mantenimiento de los logros obtenidos a lo largo del desarrollo y en la prevención de pérdidas o el reajuste o adaptación a tales pérdidas, más frecuentes en esta etapa del ciclo vital, a través de procesos de selección, optimización y compensación (Baltes, 2003). Los procesos de selección implican un reajuste de las metas personales del individuo a través de la selección de aquellas áreas de acción de máxima prioridad, como consecuencia de la anticipación o surgimiento de importantes cambios o limitaciones en los recursos personales y sociales (p.ej., una persona mayor que, ante la situación de padecimiento de una enfermedad terminal por parte de su pareja, reduce su ocio y actividad social para centrarse en el cuidado). Por su parte, los procesos de compensación entran en juego cuando se produce un cambio o una pérdida en los recursos de la persona o de su entorno, e implican emplear medios alternativos para llegar a una misma meta, a través de acciones ya presentes en el repertorio de la persona, nuevas estrategias que habrán de ser desarrolladas por la persona (p.ej., aprender estrategias para optimizar la memoria), o la utilización de medios tecnológicos concretos (ayudas protésicas: audífono para pérdidas auditivas, bastón para problemas de movilidad). La compensación también se ejerce cuando, ante las pérdidas o limitaciones para alcanzar metas concretas, la persona reestructura su sistema motivacional seleccionando nuevas metas. Finalmente, los procesos de optimización hacen referencia a la potenciación y enriquecimiento de las reservas, recursos e implicación relacionados con áreas vitales específicas; esto es, a la mejora del funcionamiento en dichas áreas concretas. Estas áreas pueden ser facetas ya desarrolladas en la vida de la persona (p.ej., invertir más tiempo y esfuerzo en las relaciones familiares) o bien ser nuevas (p.ej., descubrir o retomar aspectos relacionados con las creencias religiosas o la espiritualidad). 


\subsection{El Modelo del Desarrollo Intencio- nal}

Enmarcada dentro de la perspectiva del ciclo vital, la teoría del Desarrollo Intencional (Brandtstädter y Greve, 1994) se basa en los conceptos de voluntad, intencionalidad y orientación a metas. Plantea que el desarrollo adulto y la adaptación en la vejez dependen, de forma fundamental, de la acción de las propias personas y su capacidad para autorregularse ante los cambios y pérdidas que experimentan a lo largo de su vida, de modo que sigan activas e implicadas en metas significativas. A lo largo del ciclo vital, las personas se enfrentan a dos tareas adaptativas: a) mantener las metas personales en presencia de obstáculos importantes para su consecución, a través de actividades de tipo asimilativo o intentos activos por cambiar el entorno para conseguir las metas y objetivos personales; y b) ajustar la propia jerarquía de metas, así como los cursos de acción elegidos, ante pérdidas o restricciones irreversibles (cuando los recursos no sean suficientes o las metas sean ya inalcanzables), a través de actividades acomodativas o ajustes que las personas realizan en sus jerarquías de metas, en sus pensamientos o en sus estándares de autoevaluación ante la presencia de importantes obstáculos para la obtención de los resultados deseados. Ambos modos, asimilación y acomodación, si bien pueden parecer procesos antagónicos, en realidad se complementan mutuamente en numerosos sentidos $\mathrm{y}$, habitualmente, se coordinan para facilitar la adaptación de las personas. Numerosos problemas en la vida (p.ej., enfermedades crónicas, pérdidas afectivas o desempleo) requieren de la activación conjunta de procesos relacionados con la persistencia asimilativa y procesos de flexibilidad acomodativa. De hecho, frecuentemente, la puesta en marcha de un ajuste flexible de metas (flexibilidad acomodativa) favorece y garantiza el mantenimiento persistente del esfuerzo asimilativo.

Brandtstädter y Greve (1994) postulan que la acumulación de eventos irreversibles y la disminución de los recursos de resistencia y reserva del organismo asociados al envejecimiento hacen que, a medida que se envejece, vayan cobrando un mayor protagonismo los procesos acomodativos frente a los asimilativos. Las actividades acomodativas implican en gran medida la capacidad de desconectarse de metas no realistas o alcanzables y el reajuste de las aspiraciones personales. Para desengancharse de metas bloqueadas, diversos mecanismos pueden ponerse en marcha: relativizar la importancia de esa meta bloqueada, disminuir su atractivo, reevaluar positivamente el estado actual (p.ej, no estoy tan mal), etc. Para reevaluar positivamente el status quo, una estrategia muy útil es la consistente en cambiar (normalmente, rebajar o acercar a la realidad) los estándares de autoevaluación (p.ej., compararse con personas de su misma edad, en lugar que con otras más jóvenes) realizando, por tanto, comparaciones favorecedoras de la autoestima.

\subsection{El ajuste flexible de metas: elemento clave del envejecimiento activo y el bienestar en la vejez}

La conclusión fundamental de esta breve revisión realizada sobre los principales modelos teóricos sobre envejecimiento es la siguiente: el ser humano es un sistema orientado a metas y su implicación activa en ellas es un elemento central en su capacidad de adaptación. Esta centralidad otorgada a las metas de las personas es plenamente compartida por los modelos de personalidad más sólidamente estableci- 
dos, en los cuales la persona es considerada igualmente como un sistema orientado a metas, entendiendo como tales representaciones mentales de estados deseados a los que se aspira, y se encuentra en un proceso continuo de autorregulación (balance entre el acercamiento y alejamiento de éstas). Aunque las definiciones de "meta" pueden variar de unos modelos a otros, hay consenso en torno a la idea de que el comportamiento se organiza en torno a éstas, que serían el motor que mueve la conducta humana (Pervin, 1982). Los cambios asociados al envejecimiento implican pérdidas y limitaciones que imponen desafíos importantes a las personas al obstaculizar o imposibilitar el mantenimiento de algunas de sus metas más importantes. La capacidad de las personas para autorregularse llevando a cabo un ajuste flexible de sus metas y aspiraciones a través de procesos de selección, optimización y compensación es el ingrediente activo de la capacidad de adaptación y un requisito para el envejecimiento con éxito. De acuerdo con Rasmussen,Wrosch, Scheier, y Carver (2006), el ajuste flexible de metas implica dos procesos fundamentales: 1) desengancharse de metas inalcanzables: la persona ha de abandonar su compromiso y sus esfuerzos de acción en estas direcciones; de este modo, evita emociones negativas (p.ej., frustración) por no alcanzar dichas metas (Brandtstädter y Renner, 1990); y 2) implicarse en nuevas metas, para lo que es necesario identificar y desarrollar compromiso y esfuerzo reflejado en acciones dirigidas hacia tales metas (Wrosch, Scheier, Miller, Schulz y Carver, 2003). Este proceso contribuye al bienestar psicológico y la calidad de vida de las personas porque previene las emociones de aburrimiento, vacío o tristeza asociadas a la experiencia de no caminar en pos de ninguna meta deseada.

Existen bastantes estudios que aportan evidencia sobre los beneficios psicológicos de la capacidad para ajustar de forma flexible la jerarquía de metas personales. Duke, Leventhal, Brownlee y Leventhal (2002) encontraron que, entre las personas mayores que tuvieron que dejar de realizar actividades de ejercicio físico debido a problemas de salud, aquéllas que sustituyeron las antiguas actividades por otras nuevas presentaban un nivel superior de afecto positivo al cabo de un año que las que no lo hicieron. Por su parte, Wrosch, Miller, Scheier y Brun de Pontet (2007) encontraron que la capacidad de desengancharse de metas no alcanzables se asociaba con menores problemas de salud física, estando esta relación mediada por el nivel de sintomatología depresiva.

\section{Potenciación del ajuste flexible de metas a través de la intervención psicológica: aportaciones de la terapia cognitivo-conductual y de la terapia de aceptación y compromiso}

El ajuste flexible de metas se encuentra en constante interacción con los estados emocionales y los pensamientos de las personas. Así, la capacidad para realizar dicho ajuste puede verse profundamente interferida por variables psicológicas de las personas, relacionadas con estados o rasgos emocionales negativos (p.ej., depresión, miedos, etc.) $\mathrm{y}$ pensamientos rígidos $\mathrm{y}$ distorsionados (p.ej., absolutismo o pensamiento todonada, estereotipos, etc.) que, si no son abordados, pueden llevar a la persona a permanecer enganchada en metas no alcanzables y sin buscar unas nuevas. En este apartado se revisan las aportaciones desarrolladas en el marco de dos modelos vigentes de inter- 
vención clínica que aportan herramientas útiles para el trabajo terapéutico potenciador del ajuste flexible de metas y, por consiguiente, para potenciar el envejecimiento activo: el paradigma cognitivo-conductual y la terapia de aceptación y compromiso.

\subsection{Terapia cognitivo-conductual (TCC)}

La terapia cognitivo conductual (TCC; Beck, Rush, Shaw y Emery, 1979; Ellis, 1970), paradigma predominante en psicología clínica desde hace décadas, es una perspectiva orientada al cambio y al control que se plantea como objetivos sustituir pensamientos disfuncionales (rígidos, inflexibles) o distorsionados por otros más adaptativos y ajustados a la realidad, asumiendo que el cambio en tales cogniciones generará cambios deseables en el comportamiento, llevando a la persona a desarrollar conductas o actividades más adaptativas que facilitará una reducción de su malestar emocional. A pesar de la importancia concedida a las metas por los modelos gerontológicos y de personalidad, el abordaje de las metas y valores o aspiraciones personales de las personas, su clarificación o el compromiso con éstas como medio para incrementar su bienestar psicológico, no han sido características definitorias del protocolo de intervención TCC y no siempre son incluidos como componentes explícitos en sus paquetes de tratamiento. Más bien, en la intervención TCC se suele asumir un modelo de "normalidad" sana, representado por la ausencia de malestar emocional y la presencia de pensamientos y conductas adaptativas que, en esencia, son las mismas para todas las personas: flexibilidad de pensamiento, revaluación cognitiva ante sucesos negativos, implicación en actividades agradables, habilidades de afrontamiento, de comunicación, de asertividad y de solu- ción de problemas. La TCC suele dirigirse, por tanto, a resolver la "disfuncionalidad" que presentan los pacientes, entrenándoles en las competencias y estrategias cognitivas y conductuales y potenciando, de esta manera, su capacidad para ajustar sus metas de forma flexible a los cambios en sus circunstancias vitales. Algunas de estas estrategias serían las siguientes: a) Revaluación cognitiva: aumento del atractivo de nuevas metas o devaluación de metas no realistas; un ejemplo sería: "siempre he querido aprender otros deportes más suaves, como el golf; ahora puede ser un buen momento. Además, creo que tampoco era tan sano correr $10 \mathrm{~km}$ diarios, mis huesos sufrían mucho"; b) Procesos de comparación favorecedores de la autoestima, cambiando el estándar de referencia: "si me comparo con mi nieto de 25 años, pues claro, ¡estoy fatal!, pero mirando cómo está la gente de mi edad, ¡no estoy nada mal!; c) Educación: proporcionar información adecuada que corrija estereotipos (p.ej., "las personas mayores no pueden aprender”) y prejuicios sobre el envejecimiento para permitir el establecimiento de nuevas metas adaptativas (p.ej., aprender informática); d) Identificación y cambio de pensamientos automáticos negativos que están en la base de conductas poco adaptativas ("No salgo porque no voy a disfrutar nada"); y e) Entrenamiento en habilidades sociales, de afrontamiento y solución de problemas que se pueden presentar en el camino hacia las metas personales (p.ej., "puedo aprender informática para comunicarme con más frecuencia con mis nietos a través del correo electrónico").

Asumiendo la gran utilidad de la TCC, la experiencia clínica con personas en situaciones vitales difíciles, tales como la de afrontar el cuidado de un ser querido con 
enfermedad crónica, permite constatar que este enfoque $o$, al menos, una forma bastante extendida de aplicarlo, presenta una serie de limitaciones, entre las que pueden señalarse las siguientes (Losada y Márquez-González, 2011):

1) excesiva importancia concedida al bienestar de tipo hedónico: se busca reducir el malestar, maximizando el afecto positivo y minimizando el negativo, sin atender suficientemente al bienestar de tipo eudaimónico o satisfacción vinculada a la obtención de propósito y significado en la vida (coherencia con los valores).

2) énfasis masivo en el cambio/control de pensamientos y conductas de cara a cambiar emociones. En el envejecimiento, hay múltiples aspectos de los problemas que presentan las personas que son difícilmente modificables y la aceptación aparece como un objetivo terapéutico más conveniente en muchos casos. En este sentido, coincidimos con Petkus y Wetherell (2013) en que el énfasis de la TCC en desafiar la adecuación o validez del contenido de los pensamientos desadaptativos podría no ser tan adecuado para las personas mayores, ya que muchas de sus preocupaciones, aunque puedan ser desadaptativas y excesivas, podrían ser realistas.

3) estilo psicoeducativo y excesivamente estandarizado: en numerosas ocasiones, en TCC se funciona con una agenda preestablecida de lo que necesita la persona para normalizar su funcionamiento psicológico: pensar de forma racional, estar activa y disponer de estrategias de afrontamiento adecuadas. Esto no sería necesariamente un problema si no implicase, como ocurre en ocasiones, "imponer" a la persona, aunque sea sutilmente, ese modelo concreto de funcionamiento ideal, sin tomar el suficiente tiempo para conocer y validar la visión del mundo que tiene la persona, sus aspiraciones y sus valores personales. Esta práctica puede conducir a la resistencia al cambio de la persona, quien percibe que éste es de alguna manera "impuesto" por el experto que tiene el control de la terapia. En la actualidad, es cada vez mayor el consenso sobre la importancia central de devolver al cliente el control del proceso terapéutico, estableciendo una sólida alianza terapéutica en la que éste se sienta respetado, escuchado y aceptado en relación a sus motivaciones, creencias y formas de pensar y en la que se le anime a ser él quien decida y elija en todo momento los comportamientos a desarrollar.

\subsection{Terapia de Aceptación y Compromi- so (ACT)}

La terapia de aceptación y compromiso (ACT; Hayes et al.,1999, 2011) es la abanderada de las terapias contextuales o de tercera generación. Todas ellas comparten al menos las siguientes asunciones: a) los problemas psicológicos sólo pueden ser comprendidos y abordados en su contexto amplio, lo cual implica centrarse en la persona entendida en sentido extenso (pensamientos, emociones, valores y aspiraciones, etc.) y en interacción con el mundo y las demás personas; y b) el tratamiento adecuado de muchos problemas psicológicos pasa necesariamente por potenciar la aceptación de experiencias privadas aversivas (pensamientos y emociones) inmodificables que la persona encuentra difíciles de tolerar o asumir.

La terapia de aceptación y compromiso (ACT) resulta especialmente interesante en el contexto de reflexión generado en el presente trabajo, ya que una de sus apor- 
taciones decisivas es precisamente la formulación que plantea sobre los valores o direcciones valiosas. ACT coincide plenamente con la asunción central de los modelos gerontológicos y de personalidad sobre la importancia esencial de las metas o motivaciones de la persona, que han llegado a ser consideradas por algunos autores como el elemento definitorio del ser humano, planteando que son las metas las que dan sentido a la vida humana y que comprender a una persona significa comprender sus metas y aspiraciones (Scheier y Carver, 2001). En este sentido, una de las aportaciones fundamentales de ACT tiene que ver con la distinción que establece entre los conceptos de valor y meta/objetivo. Mientras que los valores se han definido como las direcciones vitales globales, elegidas, deseadas por las personas y que nunca llegan a alcanzarse porque uno siempre puede caminar en dirección a ese valor (como quien camina hacia el horizonte), las metas u objetivos son estados deseados específicos, que pueden alcanzarse o no y que estarían al servicio de los valores (Páez, Gutierrez, Valdivia y Luciano, 2006). ACT tiene como objetivo fundamental fomentar la clarificación de los valores y metas de la persona y el compromiso con éstos, que implica el desarrollo de acciones concretas que permiten a la persona avanzar en esa dirección. El concepto de valor como algo diferente a meta tiene especial importancia en el contexto de la gerontología y es una contribución importante a los modelos motivacionales del envejecimiento, ya que introduce un contexto en el que, ante las pérdidas y limitaciones que dificultan o imposibilitan alguna meta para las personas mayores, éstas puedan realizar un ajuste flexible de metas, renunciando a alguna de ellas y sustituyéndola por otras, sin necesidad de cambiar la dirección motivacional o valor personal al que contribuyen tales metas. Por ejemplo, un valor personal de una persona que cuida de su marido con demencia es "amarle y darle todo el cariño que pueda" y, cuando ha de tomar la decisión de ingresarle en una residencia, cambiará sus metas y acciones comprometidas con ese valor, pero el valor se mantendrá: en lugar de cuidarle en casa ahora irá a verle a la residencia y le expresará su cariño y atención con nuevas acciones, tales como participar activamente en el programa de cuidados y atención de la residencia, llevarle alimentos u objetos de su interés, etc. Los valores, fuentes de satisfacción y sentido a la vida, no tienen por qué cambiar, a pesar de que las circunstancias de las personas cambien y les impongan limitaciones que hagan que algunas metas relacionadas con ese valor no sean alcanzables. De esta forma, parece claramente justificada la relación entre los modelos teóricos sobre el envejecimiento propuestas como la terapia ACT. Así, en un reciente trabajo realizado por Alonso, López, Losada y González (2013), en el que se evalúa la eficacia de un programa de tratamiento piloto dirigido a personas mayores con dolor crónico que viven en residencias, se han combinado procedimientos basados en ACT y entrenamiento en estrategias de optimización selectiva con compensación (SOC), obteniendo resultados que avalan la eficacia de la intervención: las personas mayores que participaron se mostraron más satisfechas con el tiempo y esfuerzo que dedicaban a los aspectos que más valoraban en sus vidas.

\subsubsection{Adecuación de ACT para las personas mayores}

Se ha sugerido que el enfoque ACT podría ser especialmente apropiado para la inter- 
vención psicológica con personas mayores. Petkus y Wetherell (2013) plantean que ACT presenta una mejor acogida por parte de las personas mayores $\mathrm{y}$, en consecuencia, podría facilitar una mayor adherencia al tratamiento. Una posible razón es que ACT está interesada en las experiencias de bienestar eudaimónico, en las que la persona obtiene sentido y significado al caminar en dirección a sus valores y aspiraciones, a pesar de experimentar emociones o pensamientos incómodos o dolorosos en dicho camino hacia sus direcciones valiosas. El enfoque del bienestar eudaimónico encaja mejor con la experiencia de muchas personas mayores, quienes parecen asumir mejor que las generaciones de jóvenes el hecho de que "la vida es sufrimiento" y, a la vez, merece la pena vivirla, en virtud de los valores profundos e intrínsecos que le dan sentido y faciliten la aceptación del dolor para seguir caminando en las direcciones valiosas. Esta forma de concebir el bienestar por parte de las personas mayores parece asociarse, en muchos casos, a dificultades para entender como problemas clínicos, patológicos o anormales experiencias como la ansiedad o la depresión, para reconocer estos estados en ellos mismos y para aceptar como objetivos importantes el eliminar o disminuir tales experiencias".

En esta misma línea, al enmarcar la terapia en los valores únicos de la persona y asumir como objetivo terapéutico el que la persona consiga vivir en mayor conexión y coherencia con dichos valores personales fundamentales, ACT respeta la orientación de las personas mayores hacia valores como la generatividad o la motivación por guiar y cuidar a las nuevas generaciones (hijos, nietos) y enriquecer su relación con 1os demás (McAdams, de St. Aubin y Logan, 1993). De este modo, ACT no asume, como ocurre con frecuencia en el enfoque
TCC, un modelo de bienestar en exceso individualista.

Otro argumento propuesto por Petkus y Wetherell (2013) para sugerir la mayor idoneidad de ACT de cara a comprender los problemas psicológicos en las personas mayores es su propuesta del constructo transdiagnóstico de "evitación experiencial", definido como la tendencia a intentar controlar y/o evitar la presencia de eventos internos molestos o desagradables (emociones, pensamientos, recuerdos, sensaciones) y las circunstancias que los generan (Hayes et al., 1996). Este constructo facilita la evaluación y el tratamiento de distintos trastornos difíciles de distinguir especialmente en personas mayores ( $p$. ej., depresión y ansiedad, que, además, se dan frecuentemente de forma simultánea), ya que permite identificar la función de la conducta problemática en su contexto en lugar de la topografía concreta del comportamiento.

Previamente se señaló como limitación de la TCC su excesivo énfasis en el cambio/ control de pensamientos desajustados a la realidad (mediante la técnica de restructuración cognitiva) para reducir la frecuencia/ intensidad del malestar emocional. Las personas mayores, ante situaciones inevitables e inmodificables, presentan frecuentemente pensamientos con un impacto emocional negativo que son "realistas" y, por tanto, difícilmente pueden ser reestructurados. En este sentido, un enfoque terapéutico como ACT, que plantea como el "núcleo" de la terapia o aspecto estratégico central la aceptación de experiencias aversivas, parece especialmente adecuado para la intervención con personas que se enfrentan a situaciones difíciles inevitables asociadas a pensamientos y emociones dolorosas difíciles de cambiar. 
Además, al no basarse tanto como la TCC en estrategias terapéuticas lógico-verbalesracionales, sino en metáforas (imágenes mentales, analogías), paradojas y ejercicios experienciales, herramientas que presentan una gran capacidad para activar emociones y motivaciones en sesión, ACT podría resultar más adecuada para las personas mayores con dificultades en el pensamiento analítico o el razonamiento lógico.

\subsubsection{Evitación experiencial, bienestar y personas mayores}

Parece razonable hipotetizar que el intento por controlar experiencias internas dolorosas (pensamientos, sensaciones y emociones) en las personas mayores podría ser una barrera psicológica que impide realizar un adecuado ajuste flexible de metas, ya que paradójicamente, puede incluso aumentar los niveles de malestar a modo de "efecto boomerang" (Campbell-Sills, Barlow, Brown, y Hofmann, 2006; Rachman, 1980). La persona que presenta evitación experiencial percibe sus experiencias internas (p.ej., malestar, miedos, pensamientos concretos, frustración, etc.) como obstáculos o barreras para llevar la vida que desean llevar o, dicho de otro modo, para actuar en dirección a metas relacionadas con sus valores. Por esta razón, la evitación experiencial genera un coste importante en la vida de la persona: le aleja de sus valores y metas personales, manteniendo su insatisfacción o aumentándola (Orsillo, Roemer, y Barlow, 2003). Distintos estudios muestran una relación entre la evitación experiencial y consecuencias psicológicas negativas tanto en población general como clínica (para una revisión, ver Hayes et al., 1996).

Existen diferentes tipos de situaciones y experiencias ante las cuales algunas perso- nas mayores podrían desarrollar evitación experiencial. Algunas de éstas pueden tener que ver con dolor, rabia o frustración (entre otras emociones) ante situaciones externas inevitables, tales como pérdidas afectivas, deterioro de la salud física y disminución de la capacidad funcional, pérdida de roles (p. ej. jubilación), etc. Otras veces, la evitación puede darse en relación a pensamientos sobre el pasado, ("ya no soy el de antes"), recuerdos dolorosos, o anticipaciones del futuro ("no puedo ni pensar en cuando ya no me pueda valer por mí misma...”).

Petkus y Wetherell (2013) revisan una serie de estudios que aportan evidencia sobre los efectos adversos de la evitación experiencial sobre el bienestar psicológico de las personas mayores. Concretamente, se ha encontrado que el empleo de la estrategia de supresión del pensamiento para afrontar pensamientos incómodos se asocia con la percepción de menor sentido en la vida para personas mayores (Krause, 2007), con un mayor nivel de síntomas somáticos, depresivos y de ansiedad (Petkus, Gum y Wetherell, 2012) y con peores resultados tras el tratamiento de la depresión en personas mayores (Rosenthal, Cheavens, Compton, Thorp y Lynch, 2005). El afrontamiento evitativo parece mediar la asociación entre la exposición a traumas en el pasado y el malestar emocional en momentos posteriores de la vida (Dulin y Passmore, 2010). Además, se han encontrado asociaciones entre la evitación experiencial y la salud (relación inversa), por un lado, y la depresión y ansiedad (relaciones directas), por otro (Andrew y Dulin, 2007; Ayers et al., 2010). Por su parte, Butler y Ciarrochi (2007) encontraron una relación positiva entre la aceptación psicológica (evaluada a través de la Escala de Evitación Experien- 
cial, AAQ) y la salud objetiva, la productividad, la seguridad, la calidad de vida y el bienestar emocional en personas mayores. Estos mismos autores muestran cómo las personas mayores más satisfechas con sus vidas son aquéllas que tienen una mayor aceptación psicológica ante los cambios derivados del envejecimiento.

Parece, pues, que el enfoque ACT, dirigido a ayudar a la persona mayor a aceptar las pérdidas y limitaciones y optimizar sus recursos y fortalezas para seguir caminando en dirección a sus fuentes de significado podría ser mas apropiado que el enfoque TCC, más centrado en enseñar a la persona a cambiar su forma de percibir o interpretar sus pérdidas o limitaciones (Petkus y Wetherell, 2013). ACT se presenta como un enfoque terapéutico que puede facilitar en gran medida el ajuste flexible de metas en las personas mayores que se encuentren bloqueadas en metas inalcanzables o hayan abandonado metas importantes sin sustituirlas por nuevas metas significativas. Un manual terapéutico básico en el que se pueden encontrar numerosas herramientas terapéuticas útiles en este sentido es el libro de Wilson y Luciano (2002).

\section{Una propuesta concreta de inter- vención psicológica desde el marco de ACT: intervención con personas que cuidan a familiares mayores con demencia}

Las personas que cuidan de familiares mayores dependientes suelen presentar niveles elevados de malestar emocional (depresión, ansiedad, estrés, etc.) (para una revisión, ver Knight y Losada, 2011). Hasta la fecha, el tipo de intervención psicológica que se ha mostrado más efectiva para ayudar a estas personas es la terapia cognitivo conductual (TCC), si bien su tamaño del efecto es, en el mejor de los casos moderado, existiendo un conjunto de cuidadores que parecen no beneficiarse de este tipo de intervención.

En la situación del cuidado son muchos los aspectos difícilmente modificables, tanto externos (p.ej., diagnóstico de demencia, pérdida de memoria y de la capacidad funcional de la persona cuidada, comportamientos de agresividad y cambios de humor de sus familiares) como internos (p.ej., emociones, pensamientos, sensaciones corporales negativas del cuidador). La gran cantidad de tiempo y esfuerzo que dedican los cuidadores a ayudar a sus familiares hace que, con frecuencia, su vida se vea reducida o concentrada en torno a una única dirección valiosa: cuidar de su familiar. Habitualmente, los procesos de selección y optimización de este área de valor (cuidar) aportan un gran sentido a sus vidas y la satisfacción de hacer lo que deben y quieren hacer por su familiar. El mensaje fundamental de las intervenciones TCC, a saber, "Cuidador: cuídate a tí mismo", así como el excesivo énfasis de este tipo de terapia en el cambio de pensamientos y conductas (control), en ocasiones no encaja bien con este perfil de cuidadores. Una terapia como ACT, más centrada en la aceptación de los eventos inmodificables como parte inevitable de la vida, así como en la clarificación, toma de conciencia y compromiso con los valores personales, podría ser mejor recibida y más eficaz con esta población. Procesos psicológicos considerados nucleares desde ACT, como son la falta de aceptación de emociones y pensamientos desagradables ("por qué me tengo que sentir así de culpable, intento no pensar en lo duro que es tener que aguantar esta situación"), la falta de claridad y/o 
compromiso con otros valores importantes en sus vidas ("yo lo único que puedo hacer es cuidarle y dedicarme a él, lo demás no tengo tiempo para atenderlo"), la fusión con el pensamiento ("yo ya no puedo hacer nada para mí, se acabó mi vida”) o la falta de atención plena en el momento presente ("no salgo con amigos porque, cuando lo hago, no disfruto nada y mi cabeza está en casa, en qué estará haciendo mi madre"), podrían estar en la base del mantenimiento del malestar emocional de muchos cuidadores e interferir con su ajuste flexible de metas. Un estudio realizado con cuidadores (Spira et al., 2007) y en el que se encontró una asociación positiva entre evitación experiencial y depresión, aún controlando otras variables como el afecto negativo y los estresores, parece apoyar esta hipótesis.

En nuestro equipo de investigación consideramos una tarea de gran utilidad y relevancia diseñar y analizar la eficacia de intervenciones psicológicas dirigidas a ayudar a los cuidadores a reajustar sus metas personales a su situación actual de un modo que optimice su adaptación a dicha situación y, en este sentido, consideramos que ACT es una aproximación idónea para conseguir este objetivo. Concretamente, en la actualidad, nos encontramos aplicando dos tipos de intervención, TCC y ACT, cuya eficacia se quiere comparar entre sí y con un grupo control de mínimo contacto terapéutico. En la Tabla 1 se expone brevemente la propuesta desarrollada desde el marco de ACT, inicialmente aplicada en formato grupal en un estudio piloto (para ver una descripción detallada, ver Márquez-González et al., 2010) y que actualmente se está aplicando en formato individual.

\section{Sumario y conclusiones}

A lo largo de este trabajo se ha argumentado a favor de la hipótesis de la consistencia y complementariedad entre los modelos teóricos de envejecimiento que sustentan el paradigma del envejecimiento activo y la perspectiva de la intervención clínica, especialmente, la terapia de aceptación y compromiso (ACT). Si bien asumimos que la perspectiva cognitivo-conductual (TCC) representa un enfoque terapéutico adecuado y claramente validado en cuanto a su aplicación a personas mayores con problemas emocionales, en este trabajo se ha defendido la idea de que la intervención psicológica con personas mayores para promocionar su envejecimiento activo $\mathrm{y}$ con éxito se puede ver especialmente enriquecida y complementada por el enfoque terapéutico ACT. Concretamente, la intervención psicológica realizada desde el marco de ACT se presenta como especialmente útil para ayudar a personas mayores cuya implicación en actividades y acciones significativas se encuentra interferida o bloqueada por dificultades de tipo psicológico, relacionadas con problemas para aceptar o adaptarse a cambios importantes en sus vidas (p.ej., pérdidas) que imponen limitaciones y desafíos o, directamente, imposibilitan, el mantenimiento activo de determinados valores y de las metas asociadas a éstos. Sin duda, los procesos de ajuste flexible de metas que las personas mayores han de realizar necesariamente para adaptarse de forma adecuada a los constantes cambios vitales y continuar implicadas en actividades con significado y sentido personal, relacionadas con sus valores, se puede potenciar con herramientas terapéuticas desarrolladas desde la terapia cognitivoconductual (p.ej., potenciación de la revaluación cognitiva). Sin embargo, el enfoque ACT, más centrado en una perspectiva de bienestar eudaimónico, relacionado con la satisfacción asociada a la obtención de 
Tabla 1. Programa de intervención ACT para personas cuidadoras

\begin{tabular}{|c|c|c|}
\hline Sesión & Título de la sesión & Contenidos fundamentales \\
\hline 1 & $\begin{array}{l}\text { Identificando las } \\
\text { dificultades de acep- } \\
\text { tación y los valores }\end{array}$ & $\begin{array}{l}\text { - Establecimiento de una adecuada relación terapéutica } \\
\text { - Facilitar y validar la expresión/apertura emocional del cuidador } \\
\text { - Explorar la evitación experiencial con preguntas abiertas } \\
\text { - Evaluación de los valores del cuidador: Cuestionario narrativo de valores (B.160) }\end{array}$ \\
\hline 2 & $\begin{array}{l}\text { Creando un contexto } \\
\text { de aceptación para } \\
\text { caminar } \\
\text { hacia los valores (I) }\end{array}$ & $\begin{array}{l}\text { - Revisión de tareas para casa } \\
\text { - Trabajo en Valores: metáfora del Jardín (B;p.113) } \\
\text { - El coste de la evitación } \\
\text { - Trabajo en Aceptación como alternativa a la evitación: } \\
\text { - Conciencia emocional: Ejercicio “Contactando con mi emoción" (D.0.) } \\
\text { - Identificar el malestar "sucio" y "limpio". Ejercicio de Isabel la supercuidadora } \\
\text { (D.O.) }\end{array}$ \\
\hline
\end{tabular}

- Revisión de tareas para casa

- Trabajo en valores:

- "La planta del cuidado": elaboración del lema del cuidado (valores y metas

Creando un contexto implicados en el rol de cuidador; D.0.)

3 de aceptación para

- Metáfora del explorador en la jungla (D.0.)

caminar

- Listado de Acciones comprometidas con sus Valores

hacia los valores (II) - Trabajo en aceptación:

- Metáforas de los escaladores y el iceberg (D.0.)

- La trampa del control. Ejercicio "Elefantes rosas" (B.185)

- Atención plena: "Hojas en el Río" (B; p.216)

Análisis y afronta-

4 miento de barreras

para la acción

comprometida
- Revisión de tareas para casa

- Trabajo en valores:

- La planta de "uno mismo": ¿cómo anda de agua? Ejercicio de la mejor amiga (D.0.)

- Otros valores

- Análisis de barreras para la acción comprometida

- problemas con la organización del tiempo

- déficit en habilidades de comunicación / pedir ayuda

- barreras psicológicas: pensamientos y creencias, reglas verbales disfuncionales (fusión cognitiva)

- otras

- Trabajo en aceptación:

- ¿Aceptar o cambiar? Adaptación de la técnica de solución de problemas

- Ejercicio: invitar a una dificultad y trabajar con ella corporalmente

- Atención plena: "Escaneo corporal” (W. 148-152) 


\begin{tabular}{|c|c|c|}
\hline Sesión & Título de la sesión & Contenidos fundamentales \\
\hline 5 & $\begin{array}{l}\text { Distanciándome de } \\
\text { mi pensamiento para } \\
\text { caminar hacia mis } \\
\text { valores (I) }\end{array}$ & $\begin{array}{l}\text { - Revisión de tareas para casa } \\
\text { - Trabajo en aceptación: } \\
\text { - Metáforas: "malas hierbas" (B.113)“pasajeros en el Autobús" (B; 170), } \\
\text { - Defusión cognitiva: Ejercicio del limón (repetir hasta el absurdo "limón”; B; 218) } \\
\text { - Trabajo en valores: análisis de barreras para la acción comprometida y posibles } \\
\text { soluciones } \\
\text { - Atención plena: los pensamientos como sonidos (W.234-236) }\end{array}$ \\
\hline 6 & $\begin{array}{l}\text { Trabajando el yo } \\
\text { como contexto (I) }\end{array}$ & $\begin{array}{l}\text { - Revisión de tareas para casa } \\
\text { - Trabajo en aceptación: } \\
\text { - Exposición guiada a la emoción (Focalización) } \\
\text { - Trabajo en defusión cognitiva: } \\
\text { - Ejercicio de fisicalizar barreras (B.224) } \\
\text { - Metáfora de "radio de fondo encendida" (D.0.) } \\
\text { - Ejercicio "Saluda al pensamiento" } \\
\text { - Trabajo en Yo como contexto: } \\
\text {-Metáfora del tablero de ajedrez (B.209) } \\
\text { - Trabajo en valores: análisis de barreras para la acción comprometida y posibles } \\
\text { soluciones } \\
\text { - Atención plena: el "barómetro interno" (W. 195-197) }\end{array}$ \\
\hline 7 & $\begin{array}{l}\text { Trabajando el yo } \\
\text { como contexto (II) } \\
\text { y comienzo de la } \\
\text { recapitulación }\end{array}$ & $\begin{array}{l}\text { - Revisión de tareas para casa } \\
\text { - Trabajo en el Yo como Contexto } \\
\text { - Ejercicio del Yo Observador (B; p.213) (B; p.226) } \\
\text { - Trabajo en valores: análisis de barreras para la acción comprometida y posibles } \\
\text { soluciones } \\
\text { - Recapitulación: "mis puntos débiles" (barreras más habituales) } \\
\text { - Atención plena: "Hojas en el Río" (B; p.216) }\end{array}$ \\
\hline
\end{tabular}

$\begin{array}{ll}\text { Consolidando el } & \text { - Atención plena: "Bodyscan” (Williams et al. , 2010; pp.148-152) } \\ \text { nuevo enfoque de } & \text { - Integración de lo aprendido : metáfora de "la caja de herramientas" } \\ \text { aceptación en mi vida } & \text { (D.O.) } \\ & \text { - Evaluación de conocimientos post-intervención, satisfacción y otros aspectos } \\ & \text { cualitativos }\end{array}$

Nota:

B: manual básico de Wilson y Luciano (2002);

W: ejercicio extraído del libro de Williams, Teasdale y Segal (2010);

D.0.: ejercicio de diseño original 
propósito y significado de la propia vida, parece ser especialmente idóneo para el trabajo con personas mayores en contextos o escenarios en los que el cambio directo de las circunstancias es complicado o imposible $\mathrm{y}$, por tanto, se hace necesaria la aceptación de situaciones y/o de experiencias negativas inevitables (frustración, rabia, melancolía) para poder seguir implicado en acciones que aporten significado a la persona. El enfoque ACT, con su metodología experiencial y su estilo terapéutico marcadamente humanista y centrado en la persona, ofrece herramientas novedosas y de gran potencial terapéutico para potenciar los procesos de aceptación, de clarificación de valores y de compromiso con acciones coherentes con dichos valores.

Potenciar el envejecimiento activo implica, por definición, potenciar la activación conductual de la persona, lo cual, desde nuestra perspectiva, se puede lograr de forma más adecuada, eficaz, eficiente y duradera situando la activación del comportamiento en el marco de los valores individuales de cada persona y la adecuada aceptación de situaciones y experiencias dolorosas (p.ej., cambios o pérdidas).

Abellán, A., y Ayala, A. (2012). Un perfil de las personas mayores en España 2012. Indicadores estadísticos básicos. Madrid, Informes Portal Mayores, $n^{0}$ 131. [Fecha de publicación: 1/06/2012].

Alonso, M., López, A., Losada, A. y González, J.L. (2013). Acceptance and commitment therapy and selective optimization with compensation for older people with chronic pain. Behavioral Psychology.

Andrew, D. H. y Dulin, P. L. (2007). The relationship between self-reported health and mental health problems among older adults in New Zeland: experiential avoidance as a moderator. Aging and Mental Health, 11, 596-603.
Ayers, C., Petkus, A. J., Liu, L. Patterson, T. L., Whetherell, J. L. (2010). Negative life events and avoidant coping are associated with poorer long-term outcome in older adults treated for generalized anxiety disorder. Journal of Experimental Psycholpathology, 1, 146-154.

Baltes, P. B. y Baltes, M. M. (1990). Psychological perspectives on successful aging: The model of selective optimization with compensation. In P. B. Baltes y M. M. Baltes (Eds.), Successful aging: Perspectives from the behavioral sciences (pp. 1-34). New York: Cambridge University Press.

Baltes, P. B. (2003). Extending longevity: Dignity gain-or dignity drain? Max Planck Research.

Beck, A.T., Rush, A.J., Shaw, B.F. y Emery, G. (1979). Cognitive Therapy of Depression. New York: John Wiley y Sons.

Brandtstädter, J. y Renner, G. (1990). Tenacious goal pursuit and flexible goal adjustment: Explication and agerelated analysis of assimilative and accommodative strategies of coping. Psychology and Aging, 5, 58-67.

Brandtstadter, J. y Greve, W. (1994). The Aging Self: stabilizing and protective processes. Developmental Review, 14, 52-80.

Butler, J. y Ciarrochi, J. (2007). Psychological Acceptance and Quality of Life in the Elderly. Quality of Life Research, 16, 607-615.

Campbell-Sills, L., Barlow, D. H., Brown, T. A. y Hofmann, S. G. (2006). Effects of suppression and acceptance on emotional responses on individuals with anxiety and mood disorders. Behavior Research and Therapy, 44, 1251-1263.

Duke, J., Leventhal, H., Brownlee, S. y Leventhal, E. (2002). Giving up and replacing activities in response to illness. Journals of Gerontology Series B: Psychological Sciences and Social Sciences, 57B, 367-376.

Dulin, P. y Passmore, T. (2010). Avoidance of traumatic material mediates the relationship between accumulated lifetime trauma and late life depression and anxiety. Journal of Traumatic Stress, 23, 296-300.

Ellis, A. (1970). The essence of rational psychotherapy: A comprehensive approach to treatment. New York: Institute for Rational Living.

Fernández-Ballesteros, R., Caprara, M.G., Iñiguez, J. y García, L.F. (2004): Promoción del Envejecimiento activo: efectos del programa "Vivir con Vitalidad". Revista Española de Geriatría y Gerontología, 40, 150-161.

Gatz, M. (2007). Commentary on evidence-based psychological treatments for older adults. Psychology and Aging, 22, 52-55.

Hayes, S. C., Strosahl, K. y Wilson, K. G. (1999). Acceptance and Commitment Therapy: An experiential approach to behavior change. New York: Guilford Press. 
Hayes, S. C., Strosahl, K. y Wilson, K. G. (2011) Acceptance and commitment therapy: The process and practice of mindful change (2 $2^{\text {nd }}$ edition). New York: Guildford.

Hayes, S.C., Wilson, K.G., Gifford, E.V., Follete, V. M. y Strosahl, K. (1996). Experiential avoidance and behavior disorder: a functional dimensional approach to diagnoses and treatment. Journal of Consulting and Clinical Psychology, 64, 1.152-1.168.

Havighurst, R. J. y Albrecht, R. (1953). Older people. New York, NY: Longmans, Green.

Heckhausen, J. y Schulz, R. (1995). A life-span theory of control. Psychological Review, 102, 284-304.

Knight, B., \& Losada, A. (2011). Family Caregiving for Cognitively or Physically Frail Older Adults: Theory, Research, and Practice. In K.W. Schaie \& S.L. Willis (Eds.), Handbook of the Psychology of Aging (7th ed.)(pp. 353365). New York: Academic Press.

Krause, K. (2007). E-learning and the e-Generation: The changing face of higher education in the 21st Century. In J. Lockard y M. Pegrum (Eds.), Brave new classrooms: Educational democracy and the internet (pp.125-140). New York: Peter Lang Publishing.

Losada, A. y Márquez-González, M. (2011). Cognitive Behavioural Therapy and Acceptance and Commitment Therapy for dementia caregivers. PSIGE Newsletter, 117, 9-18.

Márquez-González, M. (2010). Nuevas herramientas para la intervención psicológica con personas mayores: la tercera generación de terapias conductuales. Revista Española de Geriatría y Gerontología, 45, 247-249.

Márquez-González, M., Romero-Moreno, R. y Losada, A. (2010). Caregiving issues in a therapeutic context: New insights from the acceptance and commintment therapy approach. In N.Pachana, K. Laidlaw y Bob Knight (Eds). Casebook of Clinical Geropsychology: International Perspectives on Practice (pp.33-53). New York: Oxford. University Press.

McAdams, D.P., de St. Aubin, E. y Logan, RL. (1993). Generativity among young, midlife, and older adults. Psychology and Aging, 8, 221-230.

Orsillo, S. M., Roemer, L. y Barlow, D. H. (2003). Integrating acceptance and mindfulness into existing cognitivebehavioral treatment for GAD: A case study. Cognitive and Behavioral Practice, 10, 223-230.

Páez-Blarrina, M., Gutiérrez-Martínez, 0., Valdivia-Salas, S. y Luciano-Soriano, C. (2006). ACT and the Importance of Personal Values in the Context of Psychological Therapy]. International Journal of Psychology and Psychological Therapy, 6, 1-20.

Pervin, L. A. (1982). The stasis and flow of behavior: Toward a theory of goals. In M. M. Page (Ed.), Nebraska Symposium on Motivation (pp. 1-53). Lincoln: University of Nebraska Press.
Petkus, A.J. y Wetherell, J.L., (2013). Acceptance and commitment therapy with older adults: Rationale and considerations, Cognitive and Behavioral Practice, 20 47-56.

Petkus, A., Gum, A., Wetherell, J. L. (2012). Thought suppression is associated with psychological distress in homebound older adults. Depression and Anxiety, 29(3), 219-225.

Rachman, S. (1980). Emotional processing. Behaviour Research and Therapy, 18, 51-60.

Rasmussen, H. N., Wrosch, C., Scheier, M. F. y Carver, C. S. (2006). Self-regulation processes and health: The importance of optimism and goal adjustment. Journal of Personality, 74, 1721-1747.

Rosenthal, M. Z., Cheavens, J. S., Compton, J. S., Thorp, S. R. y Lynch, T. R. (2005). Thought suppression and treatment outcome in late-life depression. Aging and Mental Health, 9, 35-39.

Rowe, J.W y Kahn, R.L (1997). Successful Aging. The Gerontologist, 37, 433-440.

Ryff, C. y Keyes, C. (1995). The structure of psychological well-being revisited. Journal of Personality and Social Psychology, 69, 719-727.

Scheier, M. F., Carver, C. S. y Bridges, M. W. (2001). Optimism, pessimism, and psychological well-being. In E. C. Chang (Ed.), Optimism and pessimism: Implications for theory, research, and practice (pp. 189-216). Washington, DC: American Psychological Association.

Spira, A.P., Beaudreau, S. A., Jimenez, D., Kierod, K., Cusing, M. M., Gray, H.L. y Gallagher-Thompson, D. (2007). Experiential avoidance, acceptance, and depression in dementia family caregivers. Clinical Gerontologist, 30, 55-64.

Wilson, K.G. y Luciano, M.C. (2002). Terapia de aceptación y compromiso: Un tratamiento conductual orientado a los valores. Madrid: Pirámide.

Williams, M. Teasdale, J. Segal,Z. Kabat Zinn, J (2010). Vencer la depresión: Descubre el poder de las técnicas del mindfulness. Barcelona: Paidós.

Wrosch, C., Miller, G. E., Scheier, M. F., \& Brun de Pontet, S. (2007). Giving up on unattainable goals: Benefits for health? Personality and Social Psychology Bulletin, 33, 251-265.

Wrosch, C., Scheier, M. F., Miller, G. E., Schulz, R. y Carver, C. S. (2003). Adaptive self-regulation of unattainable goals: Goal disengagement, goal reengagement, and subjective well-being. Personality and Social Psychology Bulletin, 29, 1494-1508.

Fecha de recepción: 25/06/2012

Fecha de aceptación: 11/01/2013 